\title{
Polarization analysis for the thermal chopper spectrometer TOPAS
}

\author{
Jörg Voigt ${ }^{1 \text {, a }}$, Helmut Soltner ${ }^{2}$, Earl Babcock ${ }^{1}$, Robert J. Aldus ${ }^{1}$, Zahir Salhi ${ }^{1}$, Ramil R. Gainov ${ }^{3,4}$ and Thomas Brückel ${ }^{1}$ \\ 1 Jülich Centre for Neutron Science JCNS and Peter Grünberg Institut PGI, JARA-FIT, Forschungszentrum Jülich GmbH, \\ 52425 Jülich, Germany \\ 2 Central Institute of Engineering, Electronics and Analytics, Forschungszentrum Jülich GmbH, 52425 Jülich, Germany \\ 3 Helmholtz-Zentrum Berlin für Materialien und Energie GmbH, 14109 Berlin, Germany \\ 4 Kazan Federal University, 420008 Kazan, Russia
}

\begin{abstract}
We report on the progress of the construction of the thermal time-of-flight spectrometer with polarization analysis TOPAS at the Mayer-Leibnitz Zentrum (MLZ). The instrument components approach the status to be ready for installation. The special feature of the instrument is its capability for wide-angle polarization analysis in the thermal spectral range. Here we describe a novel approach to rotate the neutron spin adiabatically into the $\mathrm{X}, \mathrm{Y}$ or $\mathrm{Z}$ direction of the laboratory frame by combination of permanent magnets aligned as Halbach rings and electrically generated fields. Despite the severe spatial restrictions the design exhibits a very high adiabaticity and interacts only weakly with the coil layout for the analyzing ${ }^{3} \mathrm{He}$ spin filter cell (SFC).
\end{abstract}

\section{Introduction}

Time-of-flight spectrometers have traditionally been used to study incoherent scattering processes, where the scattered intensity is independent of the scattering angle or varies slowly as function of momentum transfer. Typical application examples are measurements of the vibrational density of states or crystalline electric field excitations. Recent improvements in instrumentation have opened a new area of applications, namely the mapping of coherent excitations employing large pixelized detectors. For the cold energy range very powerful instruments exist both at continuous sources and at spallation sources equipped with PSD [1-5]. In the thermal energy range chopper spectrometers based at spallation sources offer routinely the opportunity to perform single crystal measurements to explore large portions of reciprocal space simultaneously [6-9]. TOPAS is a reactor-based chopper spectrometer that runs at high repetition rates to compensate the high peak flux of spallation instruments. The compact instrument design with a sample-to-detector distance $l_{\mathrm{SD}}=2.5 \mathrm{~m}$ enables repetition rates from $300 \mathrm{~Hz}$ to $900 \mathrm{~Hz}$ depending on the initial neutron wavelength $\lambda$, if an energy transfer up to $80 \%$ of the initial energy $E$ should be recorded. As a consequence the instrument is optimized for high intensity with good but not exceptional energy resolution. As the instrument resolution is mainly controlled by the chopper burst times, intensity and resolution can be traded in a very flexible way, tuning the elastic energy resolution from $3 \%$ to $10 \%$. An elliptical neutron guide effectively focuses neutrons with $10 \mathrm{meV}<E<150 \mathrm{meV}$ into a small area of $1 \times 4 \mathrm{~cm}^{2}$ yielding a high flux. TOPAS has been designed from the very beginning as an instrument, which enables polarization analysis for inelastically scattered

a e-mail: j.voigt@fz-juelich.de neutrons, e.g. to enable the determination of magnetic eigenvectors in single crystal work. So far polarization analysis has been applied in inelastic experiments mainly on three-axis spectrometers. The two instruments D7 at the ILL and DNS at MLZ offer polarization analysis combined with time-of-flight energy resolution in the cold spectral range. In both cases the neutron spin is analyzed by means of supermirror arrays. This approach is not adequate for thermal neutrons. Therefore we develop a wide-angle PA based on ${ }^{3} \mathrm{He}$ SFC. As the polarization devices reduce the flux significantly, the optimization of the instrument towards high flux is crucial.

\section{Progress on the instrument construction}

All components of the instrument are under construction and reach different levels of completion. The ${ }^{3} \mathrm{He}$ detector covering more than $15 \mathrm{~m}^{2}$ area had been procured in 2013 and was tested on the cold neutron reflectometer NOSPECS/TREFF at the MLZ to characterize the position resolution and on the hot neutron diffractometer HEIDI to calibrate the neutron sensitivity.

The construction and assembly of the spectrometer vessel was accomplished and the vacuum system was installed and tested. Running the system in automatic operation it is possible to evacuate the $75 \mathrm{~m}^{3}$ vessel overnight to cryogenic vacuum conditions, i.e. $p<10^{-5}$ mbar. The pumping time will be significantly reduced after installation of a cryopump to remove the residual water efficiently. We have also finished and tested the load lock, which enables the separation of the sample volume (300 1) from the rest of the flight path volume for the change of sample environment or samples.

This is an Open Access article distributed under the terms of the Creative Commons Attribution License 4.0, which permits unrestricted use, distribution, and reproduction in any medium, provided the original work is properly cited. 


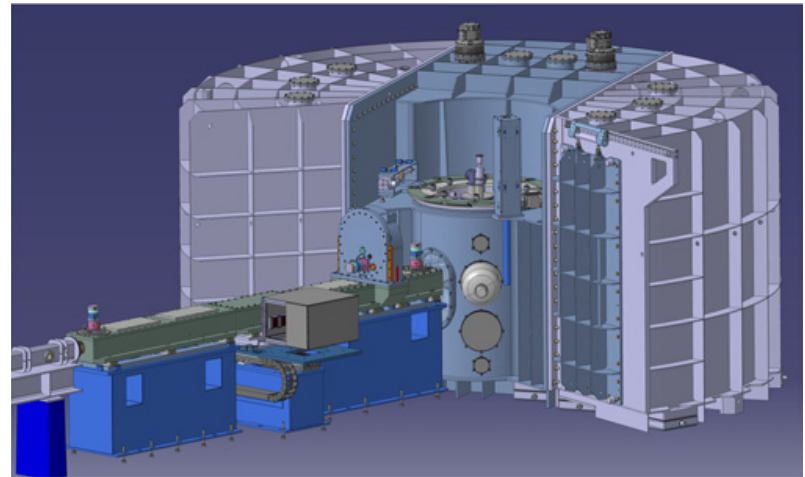

Figure 1. 3d model of the planned TOPAS spectrometer, showing the spectrometer chamber and the chopper cascade. Indicated are the positions of the neutron energy defining Fermi choppers FC1 and $\mathrm{FC} 2$ and the guide changer with the ${ }^{3} \mathrm{He}$ in-situ polarizer.

\section{Selection of the incident polarization}

Due to the high incident neutron energies $E \leq 150 \mathrm{meV}$ corresponding to $\lambda \geq 0.74 \AA$ and the convergent focused incoming beam and large area detector array, TOPAS will use ${ }^{3} \mathrm{He}$ spin filter cells for both polarization [10] and analysis [11]. The incident beam polarizer will be placed on a guide changer before the final Fermi chopper as shown in Fig. 1. From here the polarization will be maintained and guided into the PASTIS analyzer with a combination of permanent magnets and coils described in Sect. 4. This ${ }^{3} \mathrm{He}$ spin filter polarizer is based upon the experience gained with the MARIA insitu polarized ${ }^{3} \mathrm{He}$ neutron spin filter analyzer [10]. In this system a polarization of $80 \%$ was demonstrated and maintained over several days using the glass cell J1. In the subsequent years this MARIA prototype has been upgraded with improved NMR free induction decay polarization monitoring, and NMR adiabatic fast passage flipping of the ${ }^{3} \mathrm{He}$ spin, or polarization direction such that this in-situ polarized ${ }^{3} \mathrm{He}$ neutron spin filter acts simultaneously as an ideal polarization flipper. The system features a horizontal magnetic field transverse to the neutron beam using a magic box with angled plates on the side facing the sample magnet. The transverse field geometry allows for preservation of scattered neutron polarization independent of the sample field, which is vertical and ranges from $10^{-4} \mathrm{~T}$ to $1.2 \mathrm{~T}$. The current revision of the MARIA system has been used for user experiments while polarizing a $0.91{ }^{3} \mathrm{He}$ spin filter cell with two $90 \mathrm{~W}$ volume Bragg grating (VBG) narrowed diode laser array bars (DAB), see Fig. 2. During these experiments the system is stable over weeks and is used as a flipper as needed and translated in and out of the neutron beam via a motorized platform without adverse effects to the magnitude of the ${ }^{3} \mathrm{He}$ polarization. The magnitude of the ${ }^{3} \mathrm{He}$ polarization was limited to $63 \%$ during this test. However, the system will be able to provide ${ }^{3} \mathrm{He}$ polarizations of up to $75 \%$ using the same cell and lasers with proper optical optimization and replacement of two mirrors, which after the measurements were found to depolarize the laser light. With a proper optical train and optimization of parameters colleagues at NIST have shown

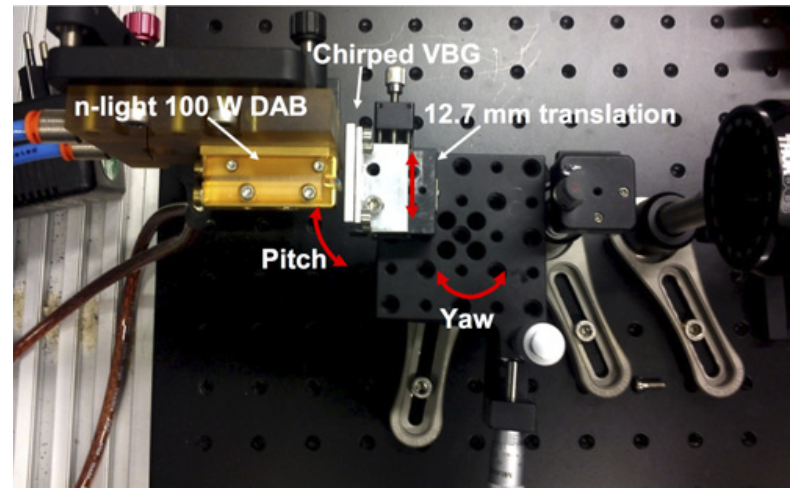

Figure 2. A photo with overlaid description of components of the new laser systems to polarize the ${ }^{3} \mathrm{He}$ using the SEOP technique. These lasers will be employed on the TOPAS ${ }^{3} \mathrm{He}$ polarization system and all JCNS ${ }^{3} \mathrm{He}$ polarization devices.

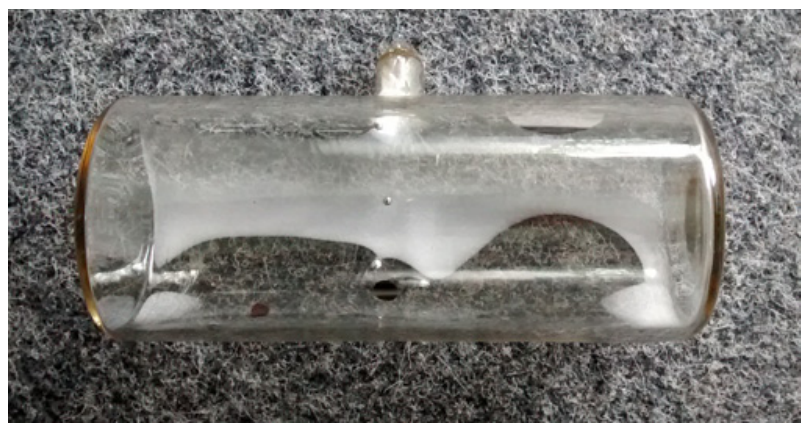

Figure 3. An example of a ${ }^{3} \mathrm{He}$ spin filter cell prepared for TOPAS. ${ }^{3} \mathrm{He}$ polarizations of up to $85 \%$ using the same laser
systems [12].

For TOPAS a copy of the components of this system will be used. The electronics, lasers, and NMR control systems will be identical to the MARIA system, and the magic-box magnetic cavity holding field will use the same concepts but have an optimized geometry for the size and wavelength of the TOPAS incident beam. The MARIA magic-box is $40 \mathrm{~cm}$ wide, $30 \mathrm{~cm}$ tall and $70 \mathrm{~cm}$ long to accept the $12.5 \mathrm{~cm}$ diameter ${ }^{3} \mathrm{He}$ cell, optics and widearea scattered neutron beam on MARIA. The TOPAS magic-box will have a simple rectangular geometry and be $27 \mathrm{~cm}$ tall, $20 \mathrm{~cm}$ wide and $50 \mathrm{~cm}$ long with a vertical field much like a $2 / 3$ scale version of the magic-box and system described in [10] but will use the upgraded volume Bragg grating narrowed lasers shown in Fig. 2 as in the current revision of the MARIA analyzer and those in [12]. The ${ }^{3} \mathrm{He}$ neutron spin filter cell will be $6 \mathrm{~cm}$ in diameter and $15 \mathrm{~cm}$ long with a ${ }^{3} \mathrm{He}$ pressure of $3 \mathrm{bar}$ corresponding to a pressure length product of $45 \mathrm{bar}-\mathrm{cm}$ for the neutrons. A picture of one such cell is shown in Fig. 3. All such cells are made of GE180 glass and filled with a hybrid potassium-rubidium alkali-metal mixture to raise the optical pumping efficiency of the SEOP process used to polarize the gas [13]. The pressure length product of this cell is chosen such that the neutron polarization for the minimum wavelength of the incident beam will be over $90 \%$ (or flipping ratio $>20$ ) for commonly achieved ${ }^{3} \mathrm{He}$ polarizations. The use of the online-polarization of the ${ }^{3} \mathrm{He}$ 


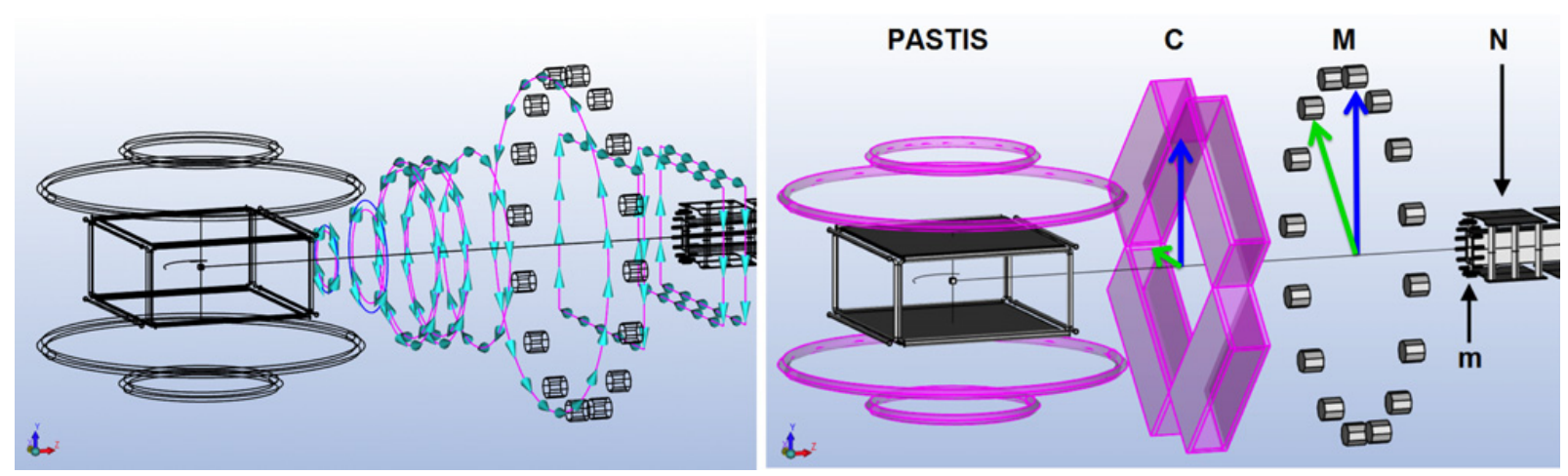

Figure 4. Sketch of the MANDHALA ring and coil arrangement for the adiabatic spin transport for the axial (X) neutron polarization (left) and the horizontal and vertical ( $\mathrm{Y}$ and $\mathrm{Z}$ ) neutron polarization (right) between the neutron guide and PASTIS. The coils are represented by line currents with the arrows indicating the current direction. The arrows at the position of the MANDHALA ring and the coil in the right figure indicate the direction of the magnetic field generated by the respective component for the case of vertical polarization (blue arrow) and horizontal polarization (green arrow).

for the incident beam will provide a better time-averaged performance as compared to ex-situ polarized SFC [14], yielding an improved data quality considering e.g. the long data collection times to record a full $S(\vec{Q}, \omega)$ map.

\section{Preparation of the incident polarization}

A major task is the spin transport from the polarizer to the PASTIS setup. A single transport setup for only one spin orientation was not deemed sufficient for our versatile experiments, but a setup which ensured an adiabatic rotation of the polarization in all three major directions, (vertical, horizontal, and axial) to match the polarization supported by PASTIS was envisaged to enable XYZ polarization analysis [15]. Thus, a system of coils and permanent magnets was designed to meet this criterion under the severe space restrictions. In the following we briefly outline the design for each of the polarization directions.

For the successful spin transport, two optimization criteria were obeyed. First, the field rotation frequency perceived by the travelling neutron should be lower than $1 / 20$ of the Larmor frequency, i.e. the adiabaticity coefficient, the ratio between the Larmor frequency and the field rotation frequency shoul be larger than 20 along the whole neutron flight path from the polarizer to the sample [16]. Second, the relative field gradient generated by the transport system at the entrance to PASTIS should be lower than $10^{-3} \mathrm{~cm}^{-1}$ to ensure a relaxation time of the polarized gas, which is determined by interactions with the cell wall or dipole dipole interactions rather than by the field gradient. Figure 4 shows a sketch of the setup for the vertical and horizontal spin orientation. In the neutron guide $(\mathrm{N})$, the guide field is realized by columns of permanent magnets, which are connected by soft iron sheets. Outside the guide, two MANDHALA (Magnetic Arrangement for Novel Discrete Halbach LAyout) rings [17] ( $\mathrm{m}$ and $\mathrm{M}$ in Fig. 4) are placed to provide the guide fields transverse to the beam direction outside of the spectrometer vessel. The ring at position $\mathrm{M}$ can be rotated to enable the adiabatic rotation from the vertical to the horizontal transverse direction. Inside the spectrometer vessel the guide field is realized by a set of rectangular coils. The respective control of the individual coil currents yields a field along the neutron path either in horizontal or in vertical direction. Their field joins then with the field created by the PASTIS coils.

For the vertical neutron polarization, both the MANDHALA ring and the four coils generate a field oriented in vertical direction. The small MANDHALA visible at the exit of the neutron guide is needed for the other polarization directions. It is not rotatable and thus adds a small horizontal field component, but which is not detrimental for the adiabatic spin transport.

The setup for horizontal spin transport looks much alike, with the only difference that the currents in the coils are switched to ensure a horizontal field in the coil and the MANDHALA $M$ takes an intermediate angular position by rotating it by $45 \mathrm{deg}$ with respect to the vertical polarization setup. The small MANDHALA $m$ at the exit of the neutron guide prepares the rotation of the neutron spin direction from vertical to horizontal.

Figure 4 shows the magnet configuration to change the neutron polarization from the vertical to the axial direction. A series of solenoids creates the magnetic field to rotate the neutron spin adiabatically into the axial direction. In this case, care has to be taken in order not to compromise the homogeneity of the PASTIS field. For this purpose, the diameter of the coils decreases on approaching PASTIS, and the coils closest to PASTIS are composed of two windings with opposed currents, as can be seen from the directions of the arrows along the circumference in this figure, in order to reduce the far fields of the coils.

The upper graph in Fig. 5 shows the main component of the above described magnet configurations for the $X, Y$ and $Z$ cases. The ratio between the Larmor frequency and the field rotation frequency, the adiabaticity coefficient, remains basically above 20 along the neutron flight path for all three cases, hence it becomes possible to transport and orient the neutron polarization vector in all three directions at the sample position. 


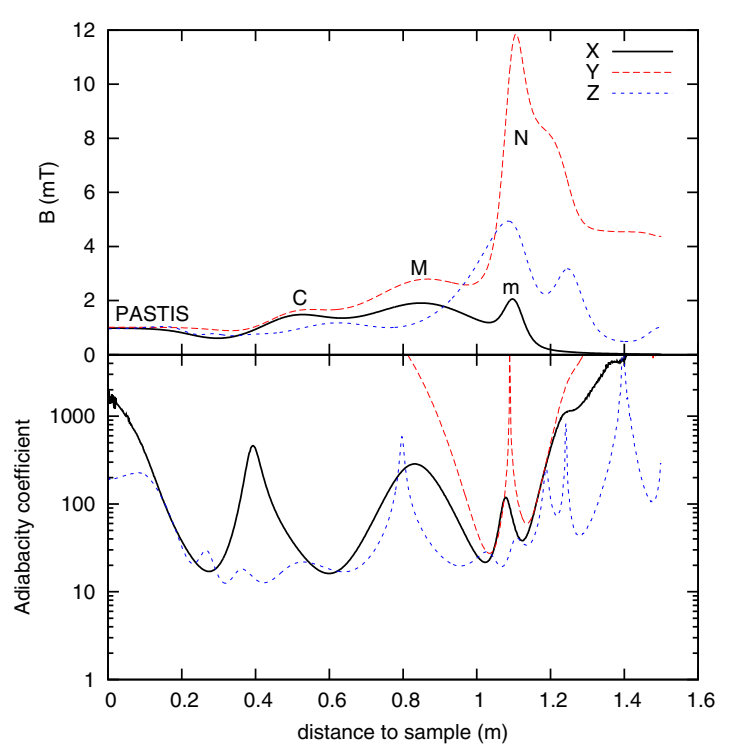

Figure 5. Upper graph: main components of the magnetic flux density for the $X, Y$ and $Z$ neutron polarization case along the neutron flight path. For the reader's orientation the peaks due to the individual components are labeled with the same letters as in Fig. 4. Lower graph: adiabaticity coefficient $\mathrm{E}$ along the neutron flight path from the end of the neutron guide to the sample for neutrons with an energy $E=167 \mathrm{meV}$.

\section{Analysis of scattered neutron polarization}

For the wide angle polarization analysis of the scattered neutrons we refine the original PASTIS design [11]. The present coil layout consists of a pair of Helmholtz coils coupled to horizontal $\mu$-metal sheets, which work as a magnetic capacitor of sorts, and generate a field in the $\mathrm{Z}$ direction. Two further pairs of rectangular coils combined with current sheets, realized by two orthogonal coils wound upon the $\mu$-metal sheets, plus magnetized iron-core solenoid rods are used for the $\mathrm{X}$ and $\mathrm{Y}$ direction. To achieve the required field homogeneity, the several elements of each orthogonal direction are currentoptimized to provide the optimal field and lowest relative field gradient over the entire volume of the SFC. The design assures relative field gradients $<10^{-3} \mathrm{~cm}^{-1}$ and large solid angle areas not interrupted by either coils or supports. In vertical direction nearly $\pm 20 \mathrm{deg}$ are open and the blind spots in the horizontal scattering plane comprise only 3 deg due to the square $X$ and $Y$ coils. For details we refer to $[18,19]$. First tests with the magic-PASTIS XYZ magnetic cavity prototype yielded reasonable ${ }^{3} \mathrm{He}$ lifetime at a low pressure for one direction. A full characterization of the system for all directions is ongoing and will be published elsewhere.

The constant initial polarization realized by the continuously pumped SEOP setup will simplify data corrections as only the time-dependent polarization of the PASTIS analysis cell will have to be corrected, and the NMR free induction decay monitoring of the polarizations of the two spin filter cells will allow us to decouple the contributions to the total neutron polarization (or flipping ratio) arising from each. Partially due to the

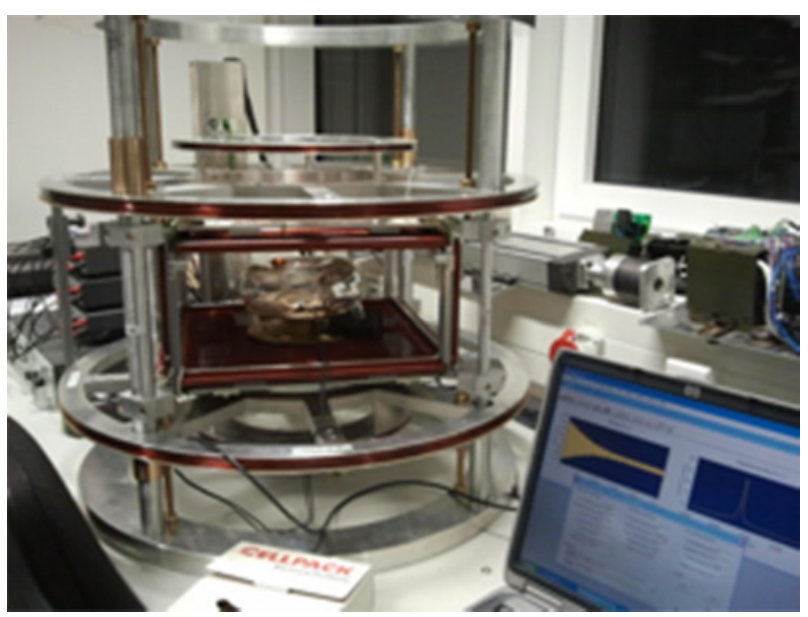

Figure 6. Photograph of the PASTIS coil setup with the torusshaped cell Homer in place.

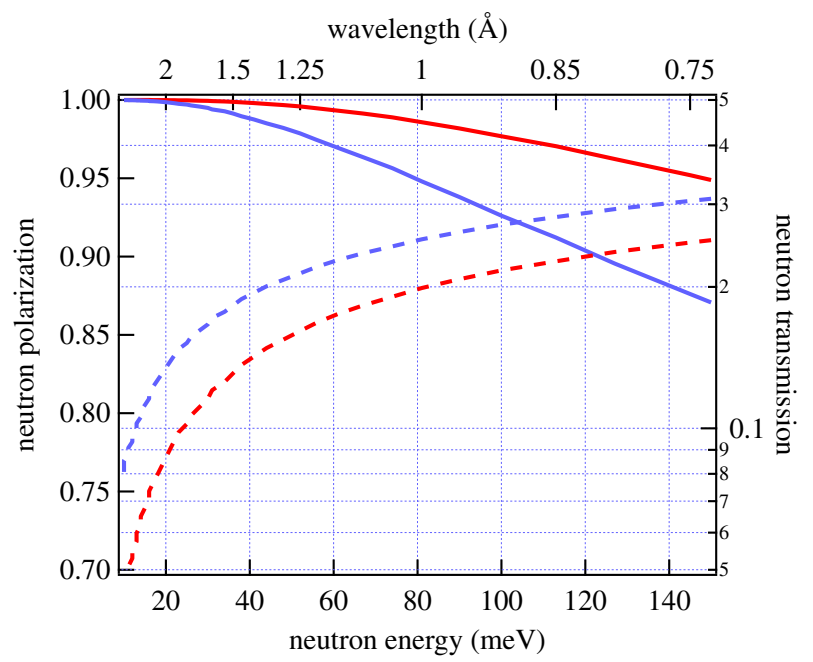

Figure 7. Calculated neutron polarization (solid lines, left axis) and neutron transmission (dashed lines, right axis) with respect to the unpolarized case as a function of neutron energy for the combination of continuously pumped SCF as polarizer and PASTIS layout with wide-angle SFC assuming $75 \%$ steady ${ }^{3} \mathrm{He}$ polarization for the polarizer and $75 \%$ initial ${ }^{3} \mathrm{He}$ polarization for the analyzer. The red/blue color corresponds to the individual polarization power of polarizer and analyzer, respectively.

size and pressure constraints, the wide-angle PASTIS analysis cell will probably contain about 3.3 bars of ${ }^{3} \mathrm{He}$ with a neutron path length of $10 \mathrm{~cm}$ corresponding to a $33 \mathrm{bar} \times \mathrm{cm}$ pressure length product. Figure 7 shows the total polarization analyzing power from the combination of both ${ }^{3} \mathrm{He}$ SFC assuming a ${ }^{3} \mathrm{He}$ polarization of $75 \%$ in both cells. Since the incident beam cell has a larger pressurelength product of $45 \mathrm{bar} \times \mathrm{cm}$ the final total neutron polarization-analysis product will be dominated by the analyzer cell. As a consequence, the data correction due to the time decay of the analysis cell should be simplified and more reliable. For example, as shown in Fig. 7 for the maximum incident neutron energy $E=150 \mathrm{meV}$, the polarizer will provide a steady polarizing power of about $95 \%$ corresponding to a flipping ratio of 39 , whereas the analysis cell should obtain an analyzing power of 
$88 \%$ or flipping ratio of 16 at the same neutron energy with its initial ${ }^{3} \mathrm{He}$ polarization. The polarizing/analyzing power of either cell will diverge to ideal values, i.e. over $98 \%$ polarization or flipping ratios of over 100 for a neutron energy $E<50 \mathrm{meV}$. For comparison, the ILL webpage states a ratio of $17 \%$ between polarized and unpolarized flux and a flipping ratio of 18 corresponding to a polarization of $89 \%$ for a neutron energy of $35 \mathrm{meV}$ on the thermal three axis spectrometer IN22, indicating that the performance now in a wide angular range can be comparable to existing polarized TAS spectrometers.

\section{Conclusion}

The construction of the TOPAS spectrometer is progressing steadily. We are now focussing on developing the necessary tools to make XYZ polarization available for the first time on a thermal chopper spectrometer. Prototypes have been developed for the polarizer and analyzer and will be optimized towards the beginning of operation at TOPAS. The novel guide field configuration is an important step to provide control of the initial neutron polarization.

\section{References}

[1] J. Ollivier, M. Plazanet, H. Schober, J. Cook, Physica B: Condensed Matter 350, 173 (2004)

[2] R. Bewley, J. Taylor, S. Bennington, Nuclear Instruments and Methods in Physics Research Section A: Accelerators, Spectrometers, Detectors and Associated Equipment 637, 128 (2011)

[3] K. Nakajima, M. Nakamura, R. Kajimoto, T. Osakabe, K. Kakurai, M. Matsuda, M. Metoki, S. Wakimoto, T. Sato, S. Itoh et al., Journal of Neutron Research 15, 13 (2007)

[4] J. Copley, J. Cook, Chemical Physics 292, 477 (2003)

[5] G. Ehlers, A.A. Podlesnyak, J.L. Niedziela, E.B. Iverson, P.E. Sokol, Review of Scientific Instruments 82, 085108 (2011)

[6] R. Kajimoto, T. Yokoo, K. Nakajima, M. Nakamura, K. Soyama, T. Ino, S. Shamoto, M. Fujita, K.
Ohoyama, H. Hiraka et al., Journal of Neutron Research 15, 5 (2007)

[7] G.E. Granroth, A.I. Kolesnikov, T.E. Sherline, J.P. Clancy, K.A. Ross, J.P.C. Ruff, B.D. Gaulin, S.E. Nagler, Journal of Physics: Conference Series 251, 012058 (2010)

[8] D.L. Abernathy, M.B. Stone, M.J. Loguillo, M.S. Lucas, O. Delaire, X. Tang, J.Y.Y. Lin, B. Fultz, Review of Scientific Instruments 83, 015114 (2012)

[9] R. Bewley, R. Eccleston, K. McEwen, S. Hayden, M. Dove, S. Bennington, J. Treadgold, R. Coleman, Physica B: Condensed Matter 385-386, Part 2, 1029 (2006)

[10] E. Babcock, S. Mattauch, A. Ioffe, Nuclear Instruments and Methods in Physics Research Section A: Accelerators, Spectrometers, Detectors and Associated Equipment 625, 43 (2011)

[11] J. Stewart, K. Andersen, E. Babcock, C. Frost, A. Hiess, D. Jullien, J. Stride, J.F. Barthélémy, F. Marchal, A. Murani et al., Physica B: Condensed Matter 385-386, 1142 (2006)

[12] W. Chen, T. Gentile, Q. Ye, T. Walker, E. Babcock, On the limits of spin-exchange optical pumping of ${ }^{3} \mathrm{He}$ (2014), submitted

[13] E. Babcock, I. Nelson, S. Kadlecek, B. Driehuys, L. Anderson, F. Hersman, T. Walker, Phys. Rev. Lett. 91, (2003)

[14] E. Babcock, A. Ioffe, Physica B: Condensed Matter 406, 2448 (2011)

[15] O. Schärpf, H. Capellmann, physica status solidi (a) 135, 359 (1993)

[16] P. Seeger, L. Daemen, Nuclear Instruments and Methods In Physics Research Section A: Accelerators Spectrometers Detectors and Associated Equipment 457, 338 (2001)

[17] H. Soltner, P. Blümler, Concepts in Magnetic Resonance Part A 36A, 211 (2010)

[18] J. Voigt, E. Babcock, T. Brückel, Journal of Physics: Conference Series 211, 012032 (2010)

[19] E. Babcock, Z. Salhi, P. Piste, G. Simeoni, A. Ioffe, Journal of Physics: Conference Series 528, 012018 (2014) 